\title{
Hereditary Leiomyomatosis and Renal Cell Carcinoma Variant of Reed's Syndrome - A Rare Case Report
}

\author{
Manas Babu, Devesh Bansal, Sony Mehta, Biju Pillai, Hariharan Krishnamoorthy* \\ *Department of Urology, Lourdes hospital, Kochi, Kerala, India \\ *dr.moorthy65@gmail.com
}

\begin{abstract}
Hereditary leiomyomatosis and renal cell carcinoma is an autosomal dominant condition associated with multiple cutaneous and uterine leiomyomas associated with renal cell carcinoma. This syndrome has been proposed to be a variant of Reed's syndrome, which is also called as multiple cutaneous and uterine leiomyomatosis. Carcinomas in this syndrome tend to be more aggressive than the normal sporadic renal cell cancers. Urologists tend to miss out these skin lesions which can act as a markers of underlying renal cell cancer. Similarly Dermatologists can ignore the screening of such patients with skin lesions for underlying renal carcinoma. In this article we present a case of hereditary leiomyomatosis and renal cell carcinoma, a variant of Reed's syndrome.
\end{abstract}

Keywords: Cutaneous Leiomyomas, Renal Cell Carcinoma, Reed's Syndrome, Uterine Leiomyomas, Heriditary Neoplasms.

\section{INTRODUCTION}

Reed's syndrome, also known as multiple cutaneous and uterine leiomyomatosis (MCUL) or multiple leiomyomatosis or leiomyomatosis cutis et uteri, was first described by Reed and colleagues in 1973. [1] This disease has autosomal dominant inheritance pattern. The syndrome is characterized by cutaneous leiomyomas in males and cutaneous leiomyomas with uterine leiomyomatosis in females. Affected females frequently develop uterine leiomyomas that are larger, more numerous and emerge earlier than those seen in the general population. A variant of Reed's syndrome associated with renal cell carcinoma is known as hereditary leiomyomatosis and renal cell carcinoma (HLRCC). [2] Both MCUL and HLRCC are caused by a heterozygous mutation in the fumarate hydratase gene (FH) located on chromosome 1q42.3-q43. [3]

\section{CASE REPORT}

We report a case of 52 year old female who presented with incidentally detected right renal mass. She had history of slowly progressive multiple painful skin nodules over back, right arm and chest region from the age of 28 years. She had undergone abdominal hysterectomy at the age of 32 for multiple uterine fibroids and menorrhagia. Histopathology report was consistent with uterine leiomyoma. Her daughter aged 26 years was recently diagnosed to have multiple uterine fibroids with menstrual irregularities but did not have skin lesions. However, other family members were devoid of any cutaneous lesions or uterine symptoms.

On examination, patient had multiple brown to pink coloured nodules and papules (Figure: 1) over her back, right arm, right shoulder and chest regions with mild local tenderness. CT Urogram ( Figure: 2) showed hypodense, contrast enhancing lesion of size $4.7 \times 3.8 \mathrm{~cm}$ at the upper pole of right kidney extending upto hilum suggestive of renal cell carcinoma (RCC). She underwent right radical nephrectomy with biopsy of the skin nodules. Histopathology showed that the renal mass to be clear cell type of RCC with papillary architecture (Figure: 3) and skin lesion were confirmed to be cutaneous leiomyomas (Figure: 4). Leiomyomas were further confirmed by immunohistochemistry (Desmin, Smooth Muscle Actin). 
Hereditary Leiomyomatosis and Renal Cell Carcinoma Variant of Reed's Syndrome - A Rare Case Report

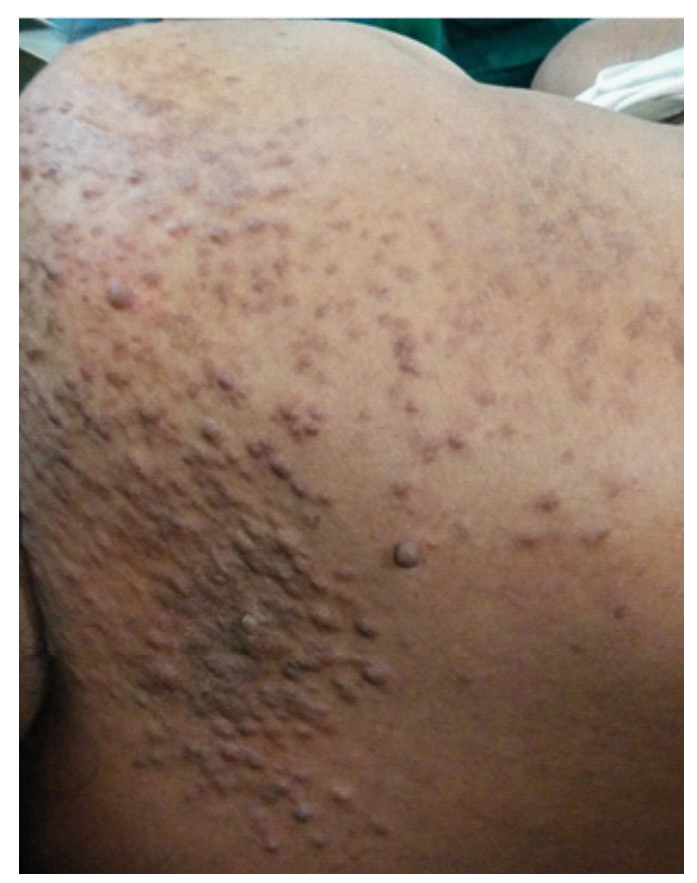

Figure 1: Multiple nodules and papules over right scapular region.

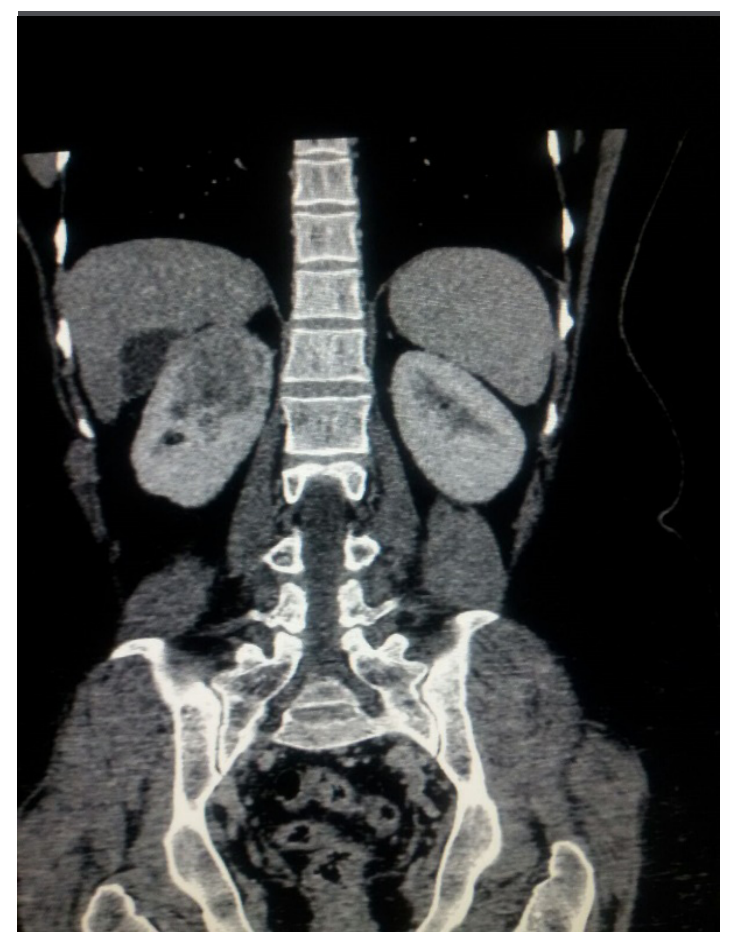

Figure 2: CT Urogram image of the right renal mass. 


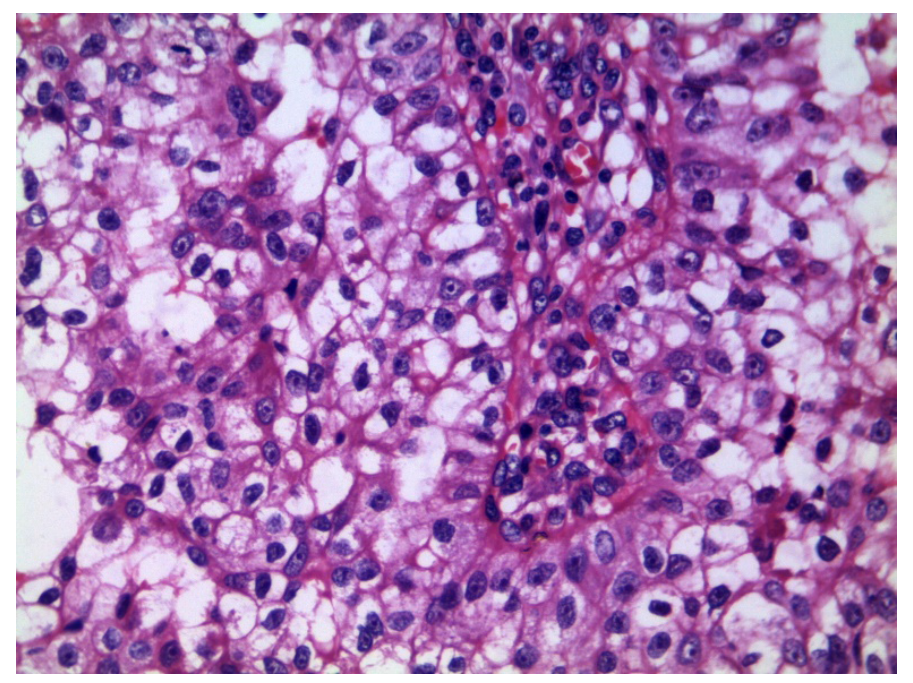

Figure 3: Microscopic picture of renal mass showing clear cell type of Renal Cell Carcinoma with papillary architecture.

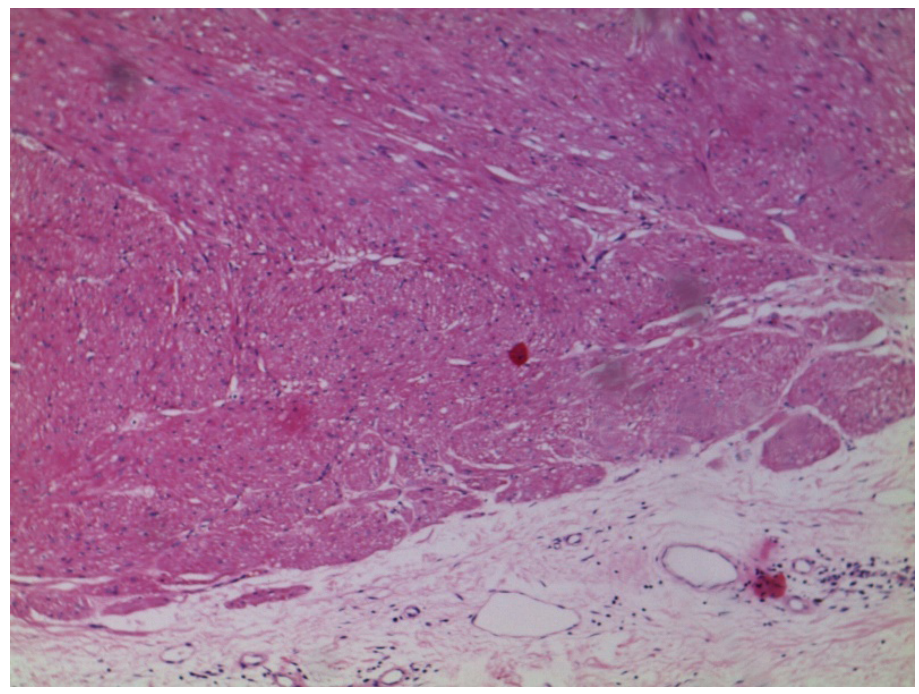

Figure 4: Microscopic picture of skin nodules consistent with leiomyomas.

Based on the clinical picture, past history of hysterectomy for uterine leiomyoma and family history, the diagnosis of hereditary leiomyomatosis and renal cell carcinoma (HLRCC) was made. Patient is on symptomatic treatment for cutaneous lesions and follow up for identifying metachronous tumour in opposite kidney for the last 3 months.

\section{DISCUSSION}

In 1854, Virchow first described cutaneous leiomyomas as a rare condition characterized by the presence of tumours, inherited in the autosomal dominant pattern. The gene defect was reported to be affecting Fumarate hydratase - one of the enzymes of the Krebs cycle. The mutation of the fumarate hydratase gene was found to result in an increased risk of development of breast cancer, urinary bladder cancer, kidney cysts and adrenal 
Hereditary Leiomyomatosis and Renal Cell Carcinoma Variant of Reed's Syndrome - A Rare Case Report

adenomas. [2] The skin lesions were described to occur equally in men and women and in 50\%, with the first lesions appearing before 20 years of age. [4] In our patient, skin lesions started occurring at the age of 28. However, these lesions were asymptomatic in earlier stages.

The pathogenesis of the pain is unclear, but Lawson et al [5] suggested that the pain results from local stimulation of the peripheral cutaneous nerves or by ischemia caused by contraction of local smooth muscles. Multiple cutaneous leiomyomas most often occur on the trunk or extremities and are typically grouped, disseminated or segmental. [6] In our patient also, lesions were grouped over right arm and over right scapular region.

Typical leiomyomas do not metastasise and the recurrence after surgical excision occurs rarely. But Barbetakis et al reported a case of metastatic nodules of leiomyosarcoma on the skin of the scalp in a patient with multiple cutaneous leiomyomatosis. This patient had undergone hysterectomy for leiomyosarcoma two years before the skin changes occurred. Hence lesions similar to leiomyomas could be malignant.

The therapy of leiomyomas includes surgical excision, cryosurgery and carbon dioxide laser ablation and a combination of pregabalin with duloxetine. [7] In our patient, the pain associated with skin lesions was not intense and therefore did not require any active intervention.

Renal cancer has low penetrance in HLRCC syndrome, with an estimated incidence from $2 \%$ to $15 \%$, and possibly as high as $32 \%$ of germline mutation affected families. [5, 6, 8, 9] Individuals affected with HLRCC are at risk for renal carcinoma as well as cutaneous and uterine leiomyomas. Female HLRCC patients frequently report a history of having large and multiple uterine fibroids at a young age, often requiring hysterectomy in their 20's or early 30's. Although the penetrance of uterine leiomyomas is very high, the risk of uterine leiomyosarcoma is very low. [5, 6, 8, 9] Wei et al [6] found that $81 \%$ of HLRCC patients had cutaneous lesions, $90 \%$ of which showed sensitivity to light touch. Unlike other hereditary renal carcinoma syndromes, HLRCC renal cancer usually presents as a solitary lesion, can be highly aggressive and can metastasize early. HLRCC renal malignancies may also have an early onset, with the youngest published metastatic patient reported at age 16. [8] These patients also develop renal cysts. Lehtonen et al [2] found $42 \%$ of mutation positive patients had renal cysts on imaging. But the malignant potential of these cysts is not known. CT and MRI are the recommended imaging modalities for the renal lesions. Considering the aggressive nature of these tumours, the affected patients should receive regular surveillance with early surgical intervention of solid lesions. The histopathology of HLRCC tumours has a characteristic morphology, demonstrating hallmark large orangiophilic nuclei and a clear perinuclear halo, with a variety of patterns such as cystic, tubulo-papillary, tubulo-solid, and are often mixed.[5,10] Our patient had solitary RCC with papillary architecture. There were no features of sarcomatous changes in the uterine leiomyoma or in the cutaneous lesions in our patient. The patient and her daughter have been kept on strict regular follow up.

Finally we conclude that, though HLRCC and Reed's syndrome are quite rare, Urologists as well as Dermatologists should be cautioned to screen patients with multiple cutaneous leiomyomas for uterine fibroids and in some cases for the presence of aggressive renal cancer.

\section{REFERENCES}

1. Reed WB, Walker R, Horowitz R. Cutaneous leiomyomata with uterine leiomyomata. Acta Derm Venereol. 1973; 53: 409-16.

2. Lehtonen $\mathrm{H}$, Kiuru M, Ylisaukko-oja S et al. Increased risk of cancer in patients with fumarate hydratase germline mutation. J Med Genet. 2006; 43: 523-6.

3. Tomlinson IP, Alam NA, Rowan AJ et al. Germline mutations in FH predispose to dominantly inherited uterine fibroids, skin leiomyomata and papillary renal cell cancer. Nat Genet. 2002; 30: 406-10. 
Hereditary Leiomyomatosis and Renal Cell Carcinoma Variant of Reed's Syndrome - A Rare Case Report

4. Kloepfer H, Krafchuk J, Derbed V, Burks J. Hereditary multiple leiomyoma of the skin. Am J Hum Genet. 1958; 10: 48-52.

5. Lawson G, Salter D, Hooper G. Angioleiomyoma of the hand. A report of 14 cases. J Hand Surg Br. 1995; 20: 479-83.

6. Wei MH, Toure 0, Glenn GM et al. Novel mutations in FH and expansion of the spectrum of phenotypes expressed in families with hereditary leiomyomatosis and renal cell cancer. J Med Genet. 2006; 43: 18-27.

7. Kostopanagiotou G, Arvaniti C, Kitsiou M et al. Successful pain relief of cutaneous leiomyomata due to Reed syndrome with the combination treatment of pregabalin and duloxetine. J Pain Symptom Manage. 2009; 38: 3-5.

8. Alam NA1, Olpin S, Leigh IM. Fumarate hydratase mutations and predisposition to cutaneous leiomyomas, uterine leiomyomas and renal cancer. Br J Dermatol. 2005; 153: 11-17.

9. Toro JR, Nickerson ML, Wei MH et al. Mutations in the fumarate hydratase gene cause hereditary leiomyomatosis and renal cell cancer in families in North America. Am J Hum Genet. 2003; 73: 95-106.

10. Merino MJ, Torres-Cabala C, Pinto P, Linehan WM. The morphologic spectrum of kidney tumors in hereditary leiomyomatosis and renal cell carcinoma (HLRCC) syndrome. Am J Surg Pathol. 2007; 31: 1578-85.

Citation: Hariharan Krishnamoorthy. "Hereditary Leiomyomatosis And Renal Cell Carcinoma: Variant Of Reed's Syndrome - A Rare Case Report". American Research Journal of Urology; 1(1): 26-30

Copyright (c) Hariharan Krishnamoorthy, This is an open access article distributed under the Creative Commons Attribution License, which permits unrestricted use, distribution, and reproduction in any medium, provided the original work is properly cited. 